\title{
Orthodontic Management of Periodontally Compromised Diabetic Individual with Missing Central Incisor
}

\section{Ambesh Kumar Rai ${ }^{*}$, Sanjay V Ganeshkar ${ }^{2}$ and Anand Patil ${ }^{2}$}

${ }^{1}$ Orthodontist, Al Reef Dental and orthodontic Centre, Ras Al khaimah, United Arab Emirates

${ }^{2}$ Department of orthodontics, SDM college of dental sciences and hospital, Dharwad, Karnataka, India

\begin{abstract}
The successful use of dental implants to replace missing teeth has become one of the most evolving areas of clinical dentistry in recent times when aesthetic dentistry is increasingly gaining prominence and treatment modality as implants are gaining acceptance as a comfortable and permanent prosthodontic solution for missing teeth especially the anterior region. This case report presents the case of a 47 year old medically compromised male with periodontal breakdown and missing central incisor treated successfully with an interdisciplinary approach to restore optimal facial aesthetics using implant supported prosthesis.
\end{abstract}

Keywords: Missing incisor; Periodontally compromised individual; Adult orthodontics; Interdisciplinary orthodontics; Ortho-perio interrelationship; Implant

\section{Introduction}

Incisors are one of the most aesthetically important teeth, and ironically are also one of the most frequently missing or lost tooth owing to trauma or periodontal breakdown. Often, patient's with missing incisor are seen covering their mouth when smiling or avoid smiling altogether in a social gathering, all in an effort to avoid the embarrassment of visible empty space in the anterior region.

The management of missing incisor is a challenge for any dentist per-se as these are the most visible teeth and even the smallest of flaw in work shouts out loud, needless to say leaving an unsatisfied patient! Often, the problem becomes compounded when the space has been edentulous for long leaving the ridge resorbed and narrowed out. Periodontal breakdown associated medical conditions, deep overbite and increased over jet, excess or inadequate space for replacement coupled with high hopes of patient's, all add to the woes of dentist often exponentially when replacing teeth in the smile zone.

While, implants have expanded restorative treatment options, treatment planning has become more complex for the dental practitioner, and an interdisciplinary team approach becomes mandatory in planning out such treatments [1-3]. This interdisciplinary approach may involve preprosthetic orthodontic treatment following consultations with an oral surgeon or periodontist and restorative dentist to ensure orthodontic alignment will facilitate the surgical, implant and restorative treatment [2].

This article presents one such case of a periodontally compromised 47 year old male diabetic and hypertensive patient who was successfully treated by an interdisciplinary approach with the help of an orthodontist, periodontist - implantologist and a prosthodontist.

\section{Chief Complaint}

A 47 year diabetic and periodontally compromised male individual was referred from the department of oral medicine and radiology to the department of orthodontics, SDM College of dental science and hospital, Dharwad, Karnataka, India. The un-aesthetics spacing in the front teeth was his chief concern and the nature of his work, which involved a lot of social interaction made him feel embarrassed especially during smiling.

\section{Case History}

A history of trauma and fracture of upper right central incisor (11) in childhood during play was reported by the patient. The tooth was not discoloured and the patient had been asymptomatic ever since. No treatment for the same was ever taken. He had developed gum problem around eight years back when spaces started to appear between the teeth, bleeding on brushing became frequent and teeth started to become and loose. Upper right central incisor was eventually extracted around five years back due to excessive mobility. The patient has been relatively free from his gum problems since three years with occasional episodes of gum bleeding.

There was a concomitant history of diabetes from 12 years and mild hypertension from past six years. The patient had been on medications for both the conditions which were well under control both by clinical and laboratory parameters.

\section{Clinical Examination}

The extraoral examination showed a convex profile with a relatively proportionate face with incisal exposure of $3 \mathrm{~mm}$ at rest. Figures 1 ( $a, b$, c, d) show patient's extraoral pictures.

Intraoral examination showed a full complement of dentition present except upper right central incisor, bilateral class I molar and canine relationship and $4 \mathrm{~mm}$ of overjet and overbite present with a matching skeletal and dental midline. Generalized gingival recession with extruded and flared out lower incisors and pathological migration of upper incisors. Figures $2(\mathrm{a}, \mathrm{b}, \mathrm{c})$ and Figures $3(\mathrm{a}, \mathrm{b})$ respectively shows patient's intraoral and occlusal pictures.

Ceplahometrically patient was a skeletal class I with hypodivergent jaw bases. IOPA (Intra Oral Periapical Radiograph) and orthopantamogram showed generalized horizontal bone loss in lower

*Corresponding author: Dr. Ambesh Kumar Rai, BDS, MDS. FAGE, Orthodontist Al Reef Dental and orthodontic Centre, Ras Al khaimah, United Arab Emirates, Tel: +971554841061; E-mail: ambeshrai@gmail.com

Received April 22, 2015; Accepted May 05, 2015; Published May 09, 2015

Citation: Rai AK, Ganeshkar SV, Patil A (2015) Orthodontic Management of Periodontally Compromised Diabetic Individual with Missing Central Incisor. Dentistry 5: 303. doi:10.4172/2161-1122.1000303

Copyright: (c) 2015 Rai AK, et al. This is an open-access article distributed under the terms of the Creative Commons Attribution License, which permits unrestricted use, distribution, and reproduction in any medium, provided the original author and source are credited. 


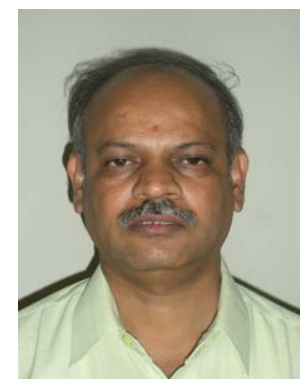

a

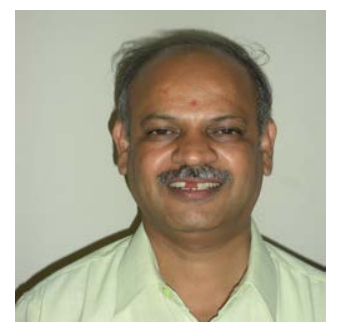

C

Figure 1 (a,b,c,d): The patient's extraoral pictures.

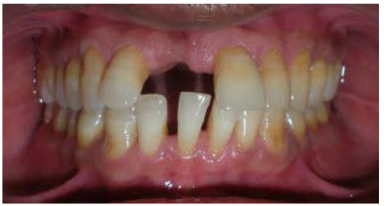

a

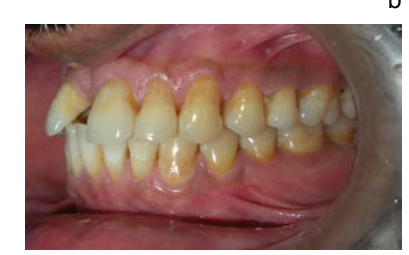

Figure $2(\mathbf{a}, \mathbf{b}, \mathbf{c})$ : The patient's intraoral pictures

anteriors which was secondary to periodontitis. Figure 4 (a, b) show patient's pre treatment X-ray pictures.

\section{Treatment Planning}

Considering the patient's concern and the aforementioned findings following treatment options were presented to the patient:

1. Elaborate orthodontic treatment with three first premolar extraction $(24,34,44)$ followed by space closure followed by conversion of lateral incisor into central with a crown and conversion of canine into lateral and premolar into canine with reshaping and restorations. Intentional root canal treatment may be needed to execute this treatment plan successfully.

2. Conventional orthodontic treatment with all first premolar extraction and retraction with maintenance of upper right central incisor (11) space and prosthetic replacement towards the end of treatment.

3. Limiting orthodontic treatment to levelling and aligning the arches. Achieving proper over jet and overbite, attaining proper root angulations, decreasing the space followed by replacement of missing teeth by Maryland Bridge/ anterior bridge work/ implant supported prosthesis.

4. No orthodontic treatment with replacement of missing tooth with a removable partial denture leaving a diastema in midline to maintain tooth proportionality with the contra lateral central incisor.

The patient opted for the third treatment plan weighing the pros and cons associated with each in option.

1-Extractions following elaborate orthodontic treatment would be time consuming and much of restorative work would still be required to achieve adequately aesthetic result.

2-The Patient was not very keen for retraction and elaborate orthodontics, besides, prosthetic replacement will still be needed.

3- Replacement with RPD (Removable Partial Denture) was no permanent solution and spaces will still be present, besides, the hassle of removing and wearing the denture.

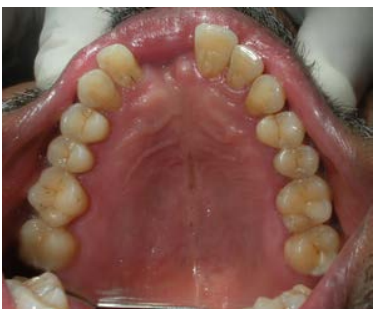

a

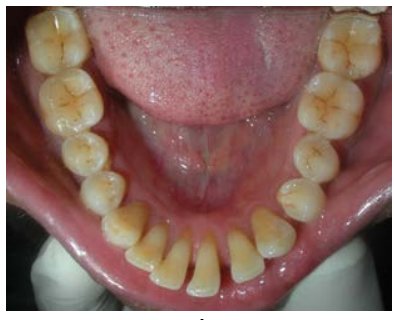

b
Figure $3(a, b)$ : The patient's occlusal pictures.

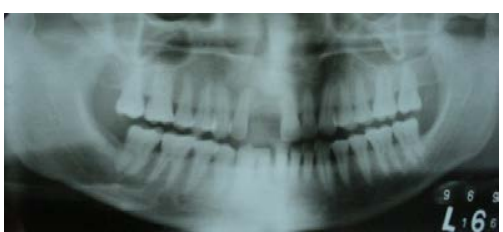

a

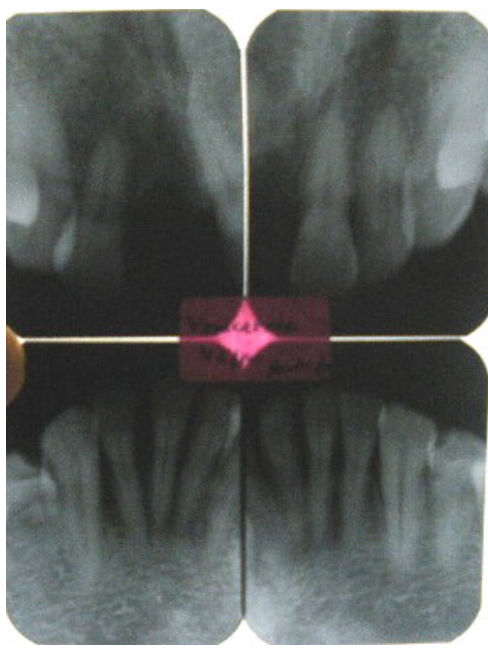

b

Figure $4(a, b)$ : The patient's pre-treatment $X$-ray pictures. 
4-Limited orthodontics with implant supported prosthesis was deemed best choice for this case.

A Maryland bridge or a six unit bridge would require crown reduction of adjacent healthy teeth, besides, getting a proper gingival aesthetics would be difficult considering the amount of anterior ridge resorption. Also, any ill fitting prosthesis may worsen the already compromised periodontal condition.

- Implants do not necessitate "altering" or "removing" parts of the natural dentition and are therefore the most conservative of the prosthodontic options for replacing missing lateral incisors $[4,5]$. Implants can also maintain the alveolar ridge, enhance occlusal function and provide optimal esthetics $[5,6]$.

An informed consent was taken in from the patient for the chosen treatment plan.

\section{Important considerations during treatment}

A) Diabetic consideration: The key to any orthodontic treatment for a patient with diabetes is good medical control. Daily rinses with a fluoride-rich mouth rinse, preferably before bedtime, has proven benefit of decreasing the chances of caries due to decreased salivation in long standing diabetes and increased plaque accumulation due to fixed appliance. Increased chances of Candida infections due to decreased salivation and high blood glucose level hence stringent monitoring is needed to rule out deterioration of the diabetic state. Also since plaque control is difficult to achieve with mechanical aids such as toothbrush and interdental brush, the use of a disinfectant mouth rinse as chlorhexidine, as an adjuvant chemical plaque control, can be considered. Diabetes-related microangiopathy can occasionally occur in the periapical vascular supply, resulting in unexplained odontalgia, percussion sensitivity, pulpitis, or even loss of vitality in sound teeth. Especially in an orthodontic treatment where forces are applied to move teeth over a significant distance, the practitioner should be alert to this phenomenon and check on a regular basis the vitality of the teeth involved. Hence light forces were to be applied so as not to overload the teeth.

Morning appointments were to be preferable. If a patient is scheduled for a long treatment as strap up or implant surgery session, which were likely to be longer than $1 \frac{1 / 2}{2}$ hours, the patient should be advised to eat their usual meal and take their medication as usual. Moreover, before the procedure starts, the dental team were check to check whether the patient has fulfilled these recommendations or not to avoid any hypoglycemic reaction in the office [7].

B) Implant consideration: To accommodate a standard implant there should be a minimum of $10 \mathrm{~mm}$ of inciso-gingival bone and a minimum of $6.0 \mathrm{~mm}$ of facial-lingual bone [8]. In cases where there is insufficient alveolar bone as in our case, ridge augmentation was necessary in addition to orthodontic repositioning of adjacent teeth to fill the bone defect [9]. Adequate space for the implant was also required between the adjacent roots $[6,10]$. The average dental implant fixture is $3.75 \mathrm{~mm}$ wide, and 1 to $2 \mathrm{~mm}$ of space is necessary between the fixture and the adjacent roots [11]. The average implant platform is $4.0 \mathrm{~mm}$ wide and requires a space of $1.0 \mathrm{~mm}$ mesially and distally between the platform and the adjacent tooth to facilitate proper healing and the development of a papilla postoperatively $[10,11]$.

C) Orthodontic consideration: Bite opening was to be done principally by "relative extrusion" i.e. by intruding the extruded and flared out lower anteriors since no ramus growth was left to compensate for true extrusive mechanics if attempted.
-One goal of orthodontic alignment was to achieve sufficient bone between the roots to place the implant. The roots of the adjacent central incisor and lateral should be parallel to slightly divergent to avoid complications resulting from root proximity. Additional mechanotherapy measures might be needed to position the roots in an implant-favourable position as bending the arch wire to accentuate root divergence.

-Aesthetics as well as occlusion must be considered in the final orthodontic positioning of the teeth adjacent to the edentulous space. To satisfy the "golden proportion" principle of aesthetics, the adjacent maxillary lateral incisor should be approximately two-thirds of the width of the central incisor $[9,11,12]$. However, if the lateral incisor is small in size, the space required to achieve symmetrical aesthetics and occlusion is primarily dictated by the width of the contra-lateral central incisor and the lateral can be build up with composite if deemed necessary.

-A riding pontic for the missing 11 to provide immediate aesthetic improvement to the patient.

\section{Treatment Progress}

Following routine periodontal maintenance procedure the treatment was first started in the lower arch to intrude the anteriors using intrusion utility arch fabricated 0.016 " NiTi followed by 0.018 " NiTi and 0.018 " stainless steel. Figure 5 shows intrusion of lower incisors using utility arch. The intrusion of extruded incisors was needed to prevent interferences in the tooth movement in the maxillary arch. The lower arch was strapped up when the lower anterior have intruded sufficiently and space closure was performed using $0.019 \times$ 0.025 " TMA wire.

A a convenience bonding of the upper arch was done followed by $0.016 " \mathrm{NiTi}, 0.018$ " NiTi for levelling and aligning the arch followed by mesiliazation of upper anterior segment using compressed coil spring on 0.020 " SS. This was followed by $0.019 \times 0.025$ " TMA for torque correction. A TMA was preferred over steel due to its low load deflection rate and decreased stiffness thus being kind to the already weakened periodontium.

A riding pontic was placed in the place of missing incisor which served dual purpose, firstly, it provided the aesthetic benefit and secondly it maintained the adequate space for the future permanent prosthesis without allowing the space to overclose.

Four months under treatment, ridge augmentation surgery was performed with autogenous bone harvested from mandibular symphysis. Figures $6(a, b, c)$ show surgical exposure of bone defect and bone graft placement. After allowing a healing phase of three months teeth movement was resumed in the grafted area to decrease the excess space before implant placement. Figures 7 (a, b, c) show intraoral photographs after bite opening and excess space closure.

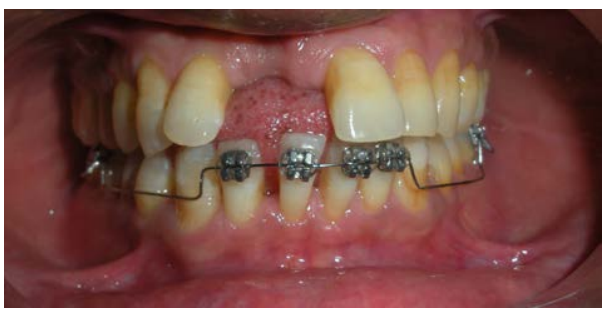

Figure 5: Intrusion of lower incisors using utility arch 


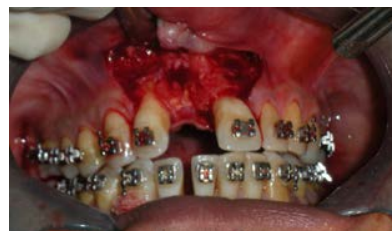

a

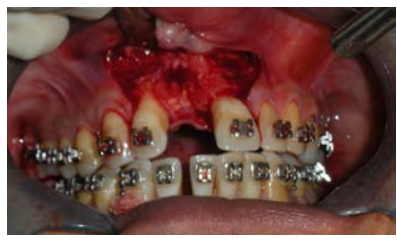

b

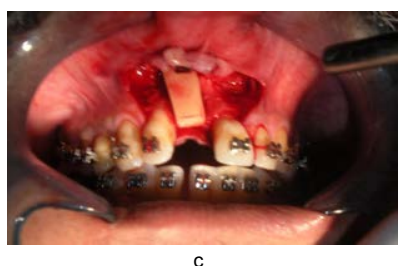

Figure $6(\mathbf{a}, \mathbf{b}, \mathbf{c})$ : Surgical exposure of bone defect and bone graft placement.

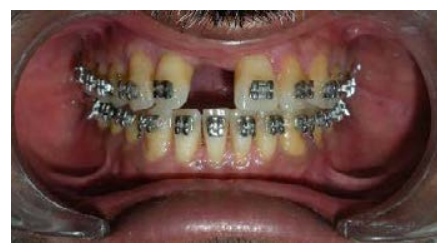

a

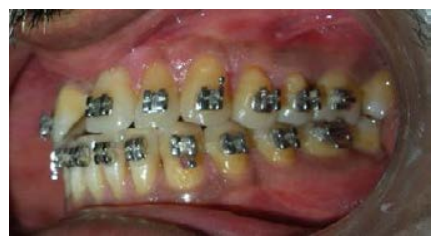

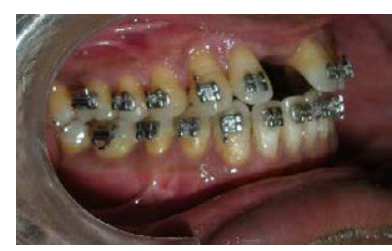

b
Figure 7 (a,b,c): Intraoral photographs after bite opening and excess space closure.

The roots of adjacent lateral incisor (12) and central incisor (22) were kept slightly divergent however, in their proper axial inclination, on the request of implantologist to facilitate implant placement in the proper direction with minimal risk of damage to the adjacent tooth root.

The implant was placed and left in place for primary integration for three months. After it was deemed satisfactorily integrated radiologically the head was exposed for supra structure construction. Figure 8 shows post implant placement radiograph.

The implant was exposed after three months of placement and was found to be adequately integrated and stable in the bone. The implant head was placed and again the site was allowed for soft tissue healing.

The orthodontic treatment continued during this time. When an adequate over jet and overbite was attained the patent was referred to the prosthodontist for the fabrication of supra structure for implant (crown for 11).

The case was debonded after the minimal residual space between the crown and the adjacent teeth were closed to establish good contact between them. Mild adjustment in torque of the upper anteriors was done to harmonize the labial prominence of teeth and the prosthetic crown. Figures $9(\mathrm{a}, \mathrm{b}, \mathrm{c}, \mathrm{d})$, Figures $10(\mathrm{a}, \mathrm{b}, \mathrm{c})$ and Figures $11(\mathrm{a}, \mathrm{b})$ show post treatment extraoral, intraoral and occlusal photographs.

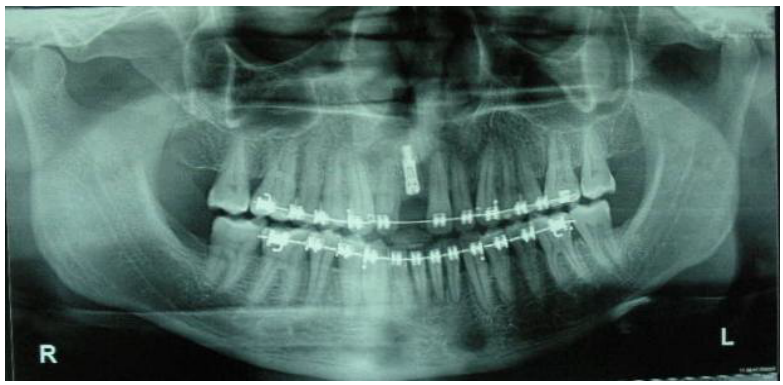

Figure 8: Post implant placement radiograph.

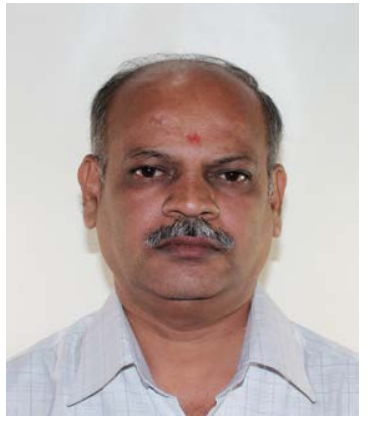

a

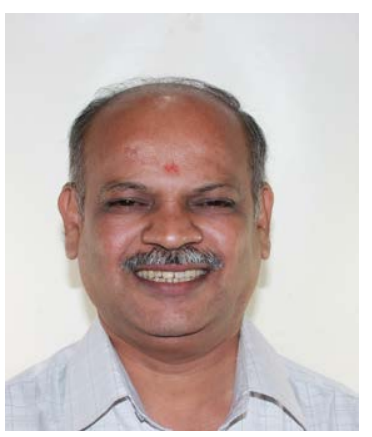

c

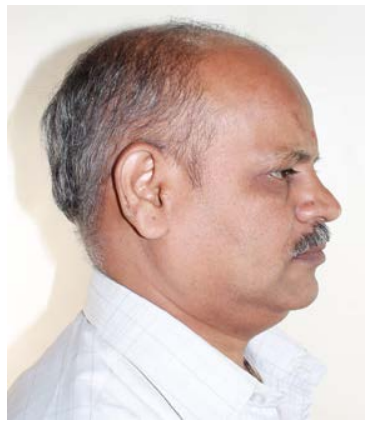

b

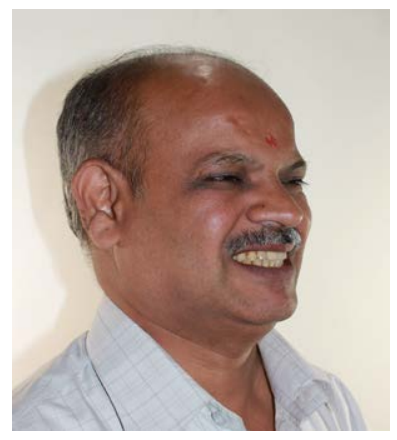

d
Figure 9 (a,b,c,d): Post treatment extraoral photographs.
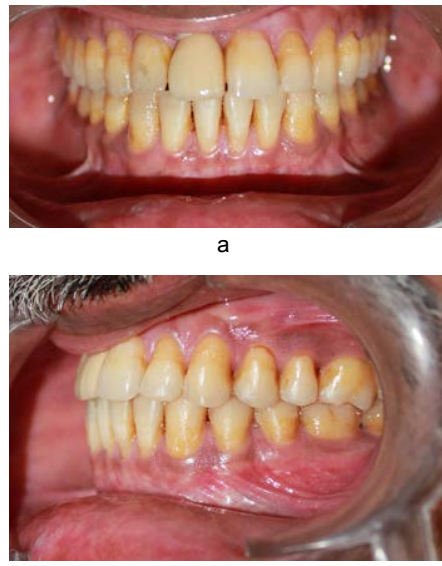

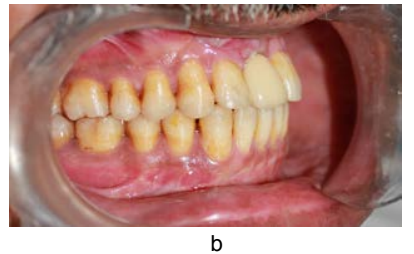

Figure $10(\mathbf{a}, \mathbf{b}, \mathbf{c})$ : Post treatment intraoral photographs. 
Citation: Rai AK, Ganeshkar SV, Patil A (2015) Orthodontic Management of Periodontally Compromised Diabetic Individual with Missing Central Incisor. Dentistry 5: 303. doi:10.4172/2161-1122.1000303

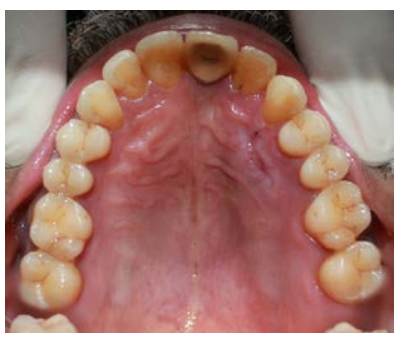

a

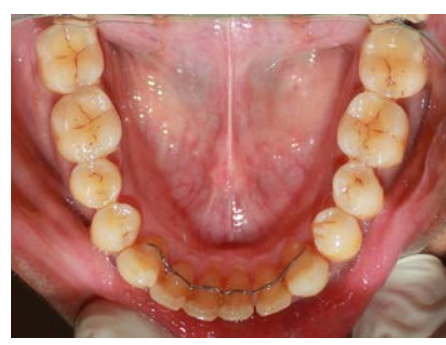

b
Figure $11(a, b)$ : Post treatment occlusal photographs.

\section{Retention}

Post treatment upper wrap around and lower canine to canine bonded retainers was placed.

\section{Conclusion}

A planned interdisciplinary approach often leads to attainment of result which is superior to that obtained by treating the patient individually by any single discipline. This case report vividly showcases one such case where the patient was presented with treatment plans which involved approach to treat him with a single discipline. Those were simple indeed, although with some compromise in result. The interdisciplinary approach used in this case was complicated and required coordination between orthodontist, periodontist, prosthodontist, oral surgeon and implantologist in the treatment execution.

Often a compromise with self ego has to be made between individuals of different speciality when planning such a case which often is the most difficult part rather than the treatment per-se, needless to say necessary and worth the effort. Finally a single key of a piano can produce a note but working in unison they produce music! The choice is always with the clinicians to take an 'I approach' and be satisfied with the note or take an extra step with a 'We approach' and create a music.

\section{References}

1. Mantzikos T, Shamus I (1998) Case report: forced eruption and implant site development. Angle Orthod 68: 179-186.

2. Schweizer CM, Schlegel KA, Rudzki-Janson I (1996) Endosseous dental implants in orthodontic therapy. Int Dent J 46: 61-68.

3. Rupp RP, Dillehay JK, Squire CF (1997) Orthodontics, prosthodontics, and periodontics: a multidisciplinary approach. Gen Dent 45: 286-289.

4. Cronin RJ, Cagna DR (1997) An update on fixed prosthodontics. J Am Dent Assoc 128: 425-436.

5. Millar BJ1, Taylor NG (1995) Lateral thinking: the management of missing upper lateral incisors. Br Dent J 179: 99-106.

6. Dialogue (1998) The role of the orthodontist on the maxillary anterior implant team. Am Assoc Orthodod 10.

7. Bensch L, Braem M, Willems G (2004) Orthodontic Considerations in the Diabetic Patient. Semin Orthod 10: 252-258.

8. Spear FM, Mathews DM, Kokich VG (1997) Interdisciplinary management of single-tooth implants. Semin Orthod 3: 45-72.

9. Shroff B, Siegel SM, Feldman S, Siegel SC (1996) Combined orthodontic and prosthetic therapy. Special considerations. Dent Clin North Am 40: 911-943.

10. Kokich VG, Spear FM (1997) Guidelines for managing the orthodonticrestorative patient. Semin Orthod 3: 3-20.

11. Richardson G, Russell KA (2001) Congenitally Missing Maxillary Lateral Incisors and Orthodontic Treatment Considerations for the Single-Tooth Implant. J Can Dent Assoc 67: 25-8.

12. Kokich V (1993) Esthetics and anterior tooth position: an orthodontic perspective. Part III: Mediolateral relationships. J Esthet Dent 5: 200-207. 